\title{
Hyperspectral imaging sustains production-process competitiveness
}

Pilar Beatriz García-Allende, Olga M. Conde, Jesús M. Mirapeix, Adolfo Cobo, and Jose M. Lopez-Higuera

A newly developed imaging system aids companies in the agri-food and industrial sectors to achieve high-speed online inspection and enhanced quality control.

Because of increased worldwide competition, efficiency improvements and establishing processes that are suitable for manufacturing high-end, high-quality products are primary commercial aims. In the agri-food industry, raw-material online characterization during the input stages of processing chains is of key importance for quality control. Classification into different quality categories and separation of unwanted spurious materials are traditionally done based on time-consuming machine-vision techniques. In industrial settings, however, welding-quality monitoring plays a major role, particularly in aeronautics and the nuclear industry, and the appearance of flaws (such as porosities or inclusions) must be prevented. Using x-rays, ultrasound, or other offline nondestructive evaluation techniques is associated with both economic and productivity costs, however.

Optical spectroscopy $(\mathrm{OS})^{1}$ has been widely used in process manufacturing, since each substance is uniquely identifiable from its spectral properties. However, traditional OS techniques generate information about the bulk properties of (or part of) a sample, ${ }^{2}$ and, in commonly used applications, many aspects of the derived sample properties result from composition heterogeneity. Imaging spectroscopy simultaneously determines the optical spectral components of a particular light-matter interaction (such as absorption) and its spatial location. It can successfully monitor sample heterogeneity. Hyperspectral imagers allow data collection in hundreds or even thousands of spectral bands. Thus, multicomponent information and high sensitivity to minor components are achieved, thus allowing enhanced quality control. Of the different types of imaging spectrometers, ${ }^{3}$

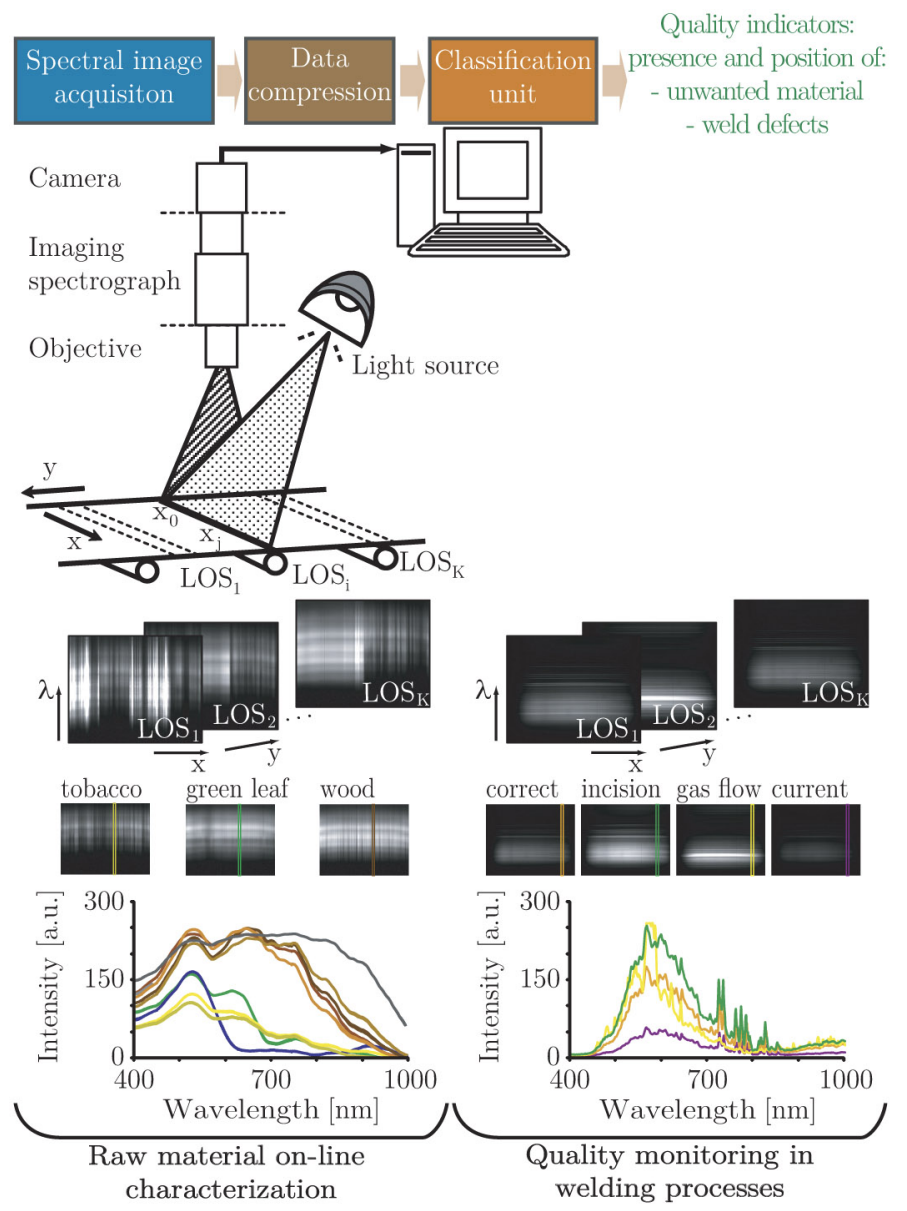

Figure 1. Global block diagram of our hyperspectral-imaging system for agri-food and industrial applications. LOS: Line of sight. a.u.: Arbitrary units. $\lambda$ : Wavelength.

the pushbroom scanner (which simultaneously scans the spectral and one spatial dimension) is particularly suitable for

Continued on next page 
industrial applications ${ }^{4}$ since conveyer-belt movement enables scanning of the other spatial dimension in a straightforward manner. These scanners allow us to obtain much more information about a given object than can be obtained from simple machine vision or single-point spectral instruments, in turn enabling a considerable increase in inspection speed.

For quality control, only the presence and position of spurious elements or weld flaws are of interest. However, hyperspectral imaging collects an immense amount of spectral information (see Figure 1). The optical spectral components of the diffuse reflectance (for raw material) or atomic emission (for welding) are measured simultaneously for every point $x_{i}$ along the line of sight by employing a prism-grating-prism imaging spectrograph ${ }^{5}$ placed between an objective lens and a monochrome camera. The movement of the conveyor belt or welding torch automatically provides the second spatial dimension. A light source, for example a halogen lamp with a quasi-flat spectrum, is required to illuminate the raw material. The datacompression block reduces both axes of the captured images. We have investigated distinct feature-extraction and selection methods ${ }^{6}$ to simultaneously extract relevant information for classification and achieve the highest possible compression.7,8

Spectral interpretation, providing material identification or flaw detection, is performed in the final stage (i.e., in the classification unit). We also considered different types of algorithms for this task. With the appropriate training, artificial neural networks (ANNs) ${ }^{9}$ proved very accurate for spuriousmaterial detection. ${ }^{10}$ This suggested that simpler (linear) algorithms could possibly also be employed. Therefore, we proposed classification units based on Fisher's (linear) discriminant analysis (FLD) ${ }^{4}$ and spectral-angle mapper (SAM). ${ }^{8}$ However, we have not been able to unambiguously identify an optimum approach. While ANNs provide the highest accuracy, FLD and SAM yield better time performance.

Figure 2 illustrates the potential of our hyperspectral system for online raw-material discrimination in the tobacco industry, where we compare the various image-analysis methodologies. (We show photographs of some of our test samples at the top.) The second and third rows show a zoom of the scene line and the spectral image, respectively. The spatial and spectral dimensions correspond to the horizontal and vertical axes, respectively. The bottom four rows display system-discrimination results based on distinct compression and classification units. The main limitation of the system is its power requirement for sufficiently bright illumination. In addition, we obtained our classification results assuming stable illumination. It is desirable to dynamically update the responsivity calibration to equalize the system's

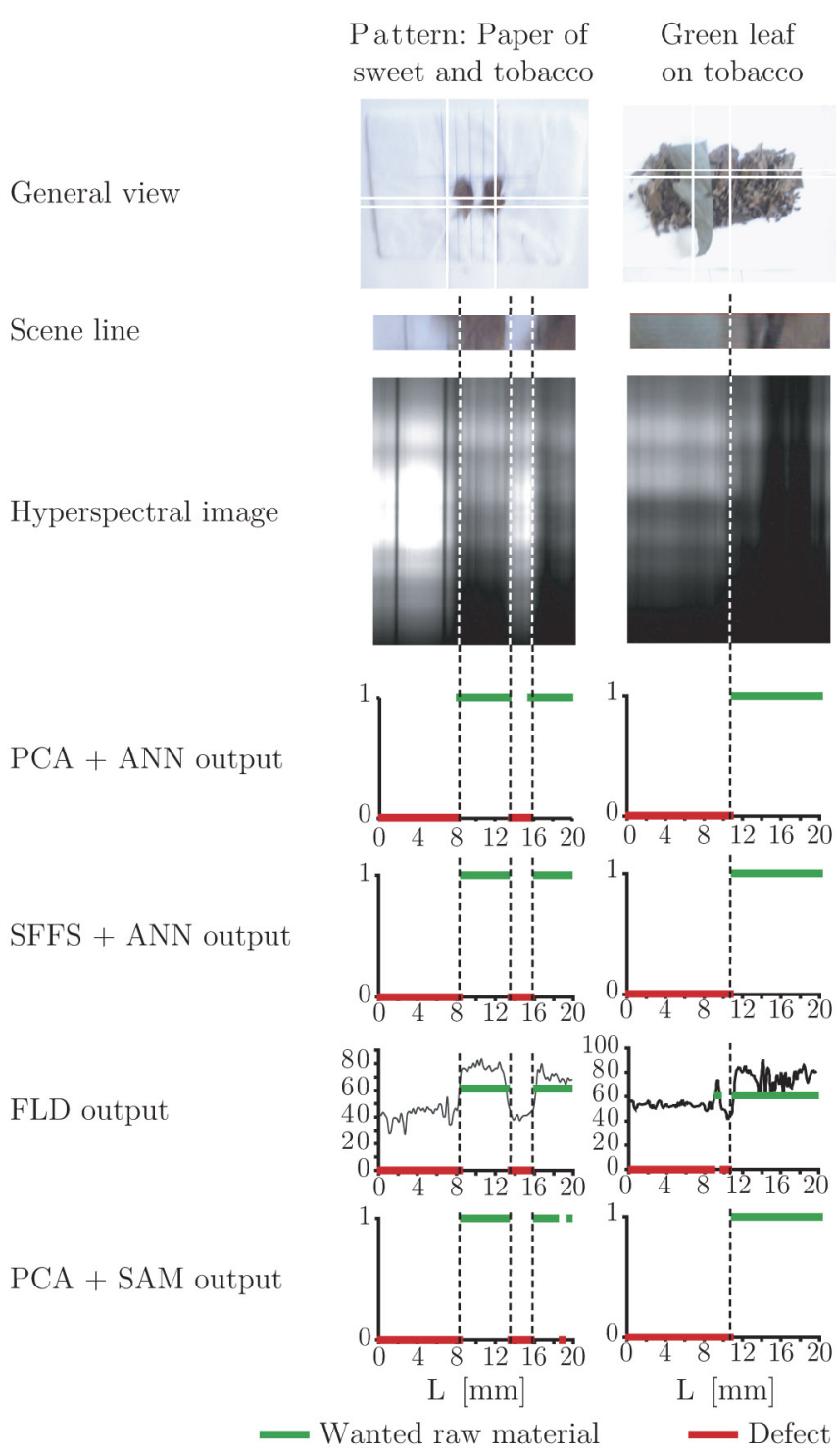

Figure 2. Raw-material discrimination offered by our newly developed hyperspectral sensor. The vertical dotted lines correlate our classification results with the hyperspectral and visible images. PCA: Principal-components analysis. ANN: Artificial neural network. SFFS: Sequential-float feature selection. FLD: Fisher's (linear) discriminant analysis. SAM: Spectral-angle mapper. L: Spatial coordinate. The vertical axes in the hyperspectral images correspond to the wavelength coordinate.

spectral response. This would allow compensation for deviations in either the illumination spectrum or the background radiation. 

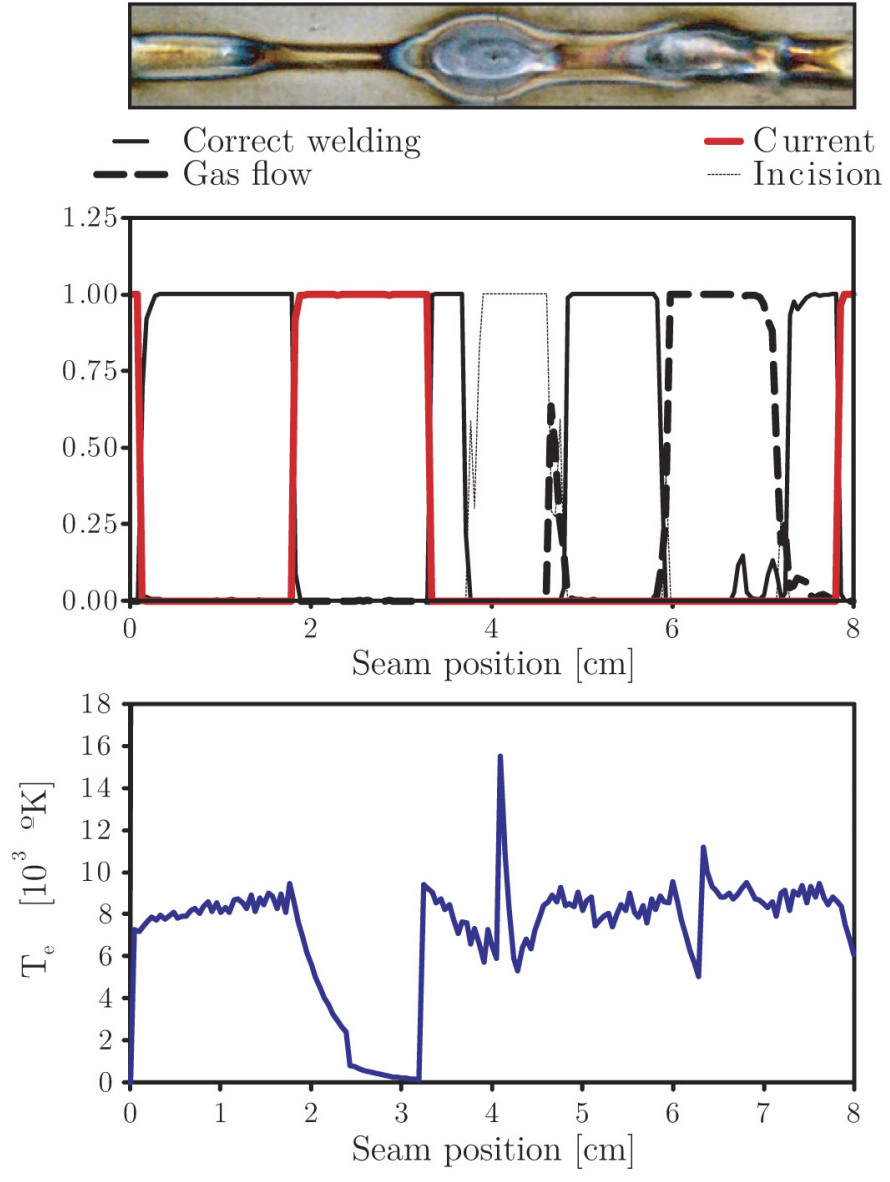

Figure 3. Comparison of hyperspectral interpretation techniques and traditional plasma spectroscopy in arc-welding defect detection and classification. The white lines delineate the LOS. $T_{e}$ : Electron temperature.

Figure 3 illustrates the process of arc-welding defect detection and classification. We have also included a comparison with the performance of traditional plasma OS as regards the sample's electron-temperature $\left(T_{\mathrm{e}}\right)$ determination. ${ }^{11,12}$ We identified a defective-weld seam section from $x \approx 1.8$ to $3.3 \mathrm{~cm}$, based on a reduction in welding current. The other two defective segments, from $x \approx 3.7$ to $4.8 \mathrm{~cm}$ and from $x \approx 6$ to $7.3 \mathrm{~cm}$, are caused by perturbations in piece thickness and shielding gas flow. The defect-detection sensitivity of our new system is similar to that of typical plasma $T_{\mathrm{e}}$ profiles.

In summary, we have illustrated the potential of hyperspectralimaging spectroscopy for industrial applications. In fact, field tests of a preliminary version of our proposed sensor for welding-quality monitoring is currently under evaluation and used to detect defects during arc welding of steam generators for nuclear plants. In addition, we are working on an extendibility study of our system to improve economic performance in the textile industry.

\section{Author Information}

Pilar Beatriz García-Allende, Olga M. Conde, Jesús M. Mirapeix, Adolfo Cobo, and Jose M. Lopez-Higuera University of Cantabria

Santander, Spain

Pilar Beatriz García-Allende received her MSc in telecommunications engineering in 2005 and is currently pursuing a PhD in the Photonics Engineering group. Her research interests focus on developing interpretation techniques and devices based on imaging spectroscopy for commercial, industrial, and medical applications.

Olga Conde received her ME and PhD degrees in telecommunications (in 1994 and 1999, respectively) from the University of Cantabria, where she is currently a faculty member in the Photonics Engineering group. Her interests focus on processing tools for OS data, light-tissue interaction modeling, and spectroscopic-system development for industrial applications.

Jesús Mirapeix received his $\mathrm{ME}$ and $\mathrm{PhD}$ degrees in telecommunications from the University of Cantabria in 2000 and 2007, respectively. His current research in the Photonics Engineering group focuses on plasma spectroscopy applied to real-time online welding-quality assurance.

Adolfo Cobo received his ME and PhD degrees in telecommunications from the University of Cantabria in 1994 and 1999, respectively. His teaching and research activities are related to optical communications and sensors for industrial applications. He has co-authored more than 100 papers in journals and conference proceedings, as well as eight patent applications.

Jose Lopez Higuera received his $\mathrm{ME}$ and $\mathrm{PhD}$ degrees in telecommunications from the Technical University of Madrid (Spain). He leads the Photonics Engineering group and is presently developing photonics instrumentation and opticalfiber sensor systems. He has co-authored more than 300 publications and obtained several patents. 
References

1. W. Schmidt, Optical spectroscopy in chemistry and life sciences, Wiley-VCH, 2005.

2. S. J. Millar, M. B. Whitworth, A. Chau, and J. R. Gilchrist, Mapping food composition using NIR hyperspectral imaging, New Food 3, pp. 34-39, 2008.

3. M. Aikio, Hyperspectral prism-grating-prism imaging spectrograph, $\mathrm{PhD}$ thesis, VTT Technical Research Center-Electronics, 2001.

4. P. B. García-Allende, O. M. Conde, J. Mirapeix, A. Cobo, and J. M. Lopez-Higuera, Quality control of industrial processes by combining a hyperspectral sensor and Fisher's linear discriminant analysis, Sens. Actuat. B-Chem. 129 (2), pp. 977-984, 2008.

5. T. Vaarala, A. Aikio, and H. Keraenen, An advanced prism-grating-prism imaging spectrograph in on-line industrial applications, Proc. SPIE 3101, pp. 322-330, 1997.

6. L. Gomez-Chova, L. Calpe, G. Camps-Valls, J. D. Martin, E. Soria, J. Vila,

L. Alonso-Corda, and J. Moreno, Feature selection of hyperspectral data through local correlation and SFFS for crop classification, IEEE Int'l Geosci. Remote Sens. Symp. Proc. 1, pp. 555-557, 2003.

7. O. M. Conde, M. Amado, P. B. García-Allende, A. Cobo, and J. M. LopezHiguera, Evaluation of PCA dimensionality reduction techniques in imaging spectroscopy for foreign object detection, Proc. SPIE 6565, p. 65650M, 2007.

8. P. B. García-Allende, O. M. Conde, J. Mirapeix, A. M. Cubillas, and J. M. Lopez-Higuera, Data processing method applying principal component analysis and spectral angle mapper for imaging spectroscopic sensors, IEEE Sens. J. 8 (7), pp. 1310-1316, 2008.

9. C. H. Chen, Fuzzy logic and neural network handbook, McGraw Hill, 1996

10. P. B. García-Allende, O. M. Conde, A. M. Cubillas, C. Jauregui, and J. M. Lopez-Higuera, New raw material discrimination system based on a spatial optical spectroscopy technique, Sens. Actuat. A-Phys. 135 (2), pp. 605-12, 2007.

11. A. Ancona, V. Spagnolo, P. M. Lugara, and M. Ferrara, Optical sensor for real-time monitoring of $\mathrm{CO}_{2}$ laser welding process, Appl. Opt. 40, pp. 6019-6025, 2001.

12. J. Mirapeix, A. Cobo, O. M. Conde, C. Jauregui, and J. M. Lopez-Higuera, Fast algorithm for spectral processing with application to on-line welding quality assurance, Meas. Sci. Technol. 17 (10), pp. 2623-2629, 2006. 